\section{$\underset{\substack{\text { hommes } \\ \text { \& migrations }}}{ }$}

\section{Hommes \& migrations}

Revue française de référence sur les dynamiques

migratoires

$1314 \mid 2016$

Migrations chinoises et générations

\title{
Grandir dans une communauté commerçante
}

Les choix professionnels de jeunes issus de familles d'entrepreneurs wenzhou

\section{Ya-Han Chuang}

\section{(2) OpenEdition}

\section{Journals}

Édition électronique

URL : http://journals.openedition.org/hommesmigrations/3636

DOI : 10.4000/hommesmigrations.3636

ISSN : 2262-3353

Éditeur

Musée national de l'histoire de l'immigration

Édition imprimée

Date de publication : 1 avril 2016

Pagination : 53-61

ISBN : 978-2-919040-35-3

ISSN : $1142-852 X$

Référence électronique

Ya-Han Chuang, "Grandir dans une communauté commerçante », Hommes \& migrations [En ligne], 1314 | 2016, mis en ligne le 01 avril 2019, consulté le 19 avril 2019. URL : http://

journals.openedition.org/hommesmigrations/3636 ; DOI : 10.4000/hommesmigrations.3636 


\title{
GRANDIR DANS \\ UNE COMMUNAUTÉ \\ COMMERÇANTE
}

LES CHOIX PROFESSIONNELS DE JEUNES ISSUS DE FAMILLES D'ENTREPRENEURS WENZHOU

par YA-HAN CHUANG, post-doctorante associée au Groupe d'études des méthodes d'analyse sociologique de la Sorbonne (GEMASS).

\author{
L'esprit d'entreprise et l'art du commerce représentent \\ deux traits caractéristiques de la communauté wenzhou. \\ Forts de ce capital culturel fondé sur la performance \\ économique, les enfants de commerçants semblent partagés \\ entre la poursuite de longues études et la reprise des affaires \\ de leurs parents. Ces jeunes wenzhou ont à rester fidèles aux \\ valeurs entrepreneuriales transmises tout en s'émancipant. \\ Aussi, ne ménagent-ils pas leurs efforts pour sortir du modèle \\ traditionnel des petites entreprises familiales et incarner \\ les relais transnationaux d'une économie mondialisée.
}

En France, depuis une trentaine d'années, les migrants chinois originaires de la région de Wenzhou, région réputée pour sa tradition artisanale et pour le nombre important de très petites entreprises familiales ${ }^{1}$, deviennent des acteurs économiques importants. En plus des secteurs d'activité traditionnellement occupés par les migrants en Europe - la restauration, le textile, la maroquinerie, etc. -, ils ont établi un énorme marché de commerce de gros d'import-export qui compte plus de mille magasins dans la banlieue parisienne ${ }^{2}$. L'objectif de cet article est d'interroger l'influence qu'a le fait de grandir dans une famille d'entrepreneurs sur les choix professionnels des jeunes issus de l'immigration. Ce travail cible plus particulièrement les jeunes issus de familles wenzhou ${ }^{3}$, nés ou ayant grandi en France ${ }^{4}$. Comme l'a constaté la théorie de l'assimilation segmentée, les trajectoires individuelles de l'assimilation et de l'acculturation des descendants d'immigrés sont souvent encadrées,

1. Kristen Parris, "Local initiative and national reform: The Wenzhou model of development", in The China Quarterly, vol. 134, 1993, pp. 242-263; Richard Beraha, Junliang Pan, Liwen Dong et al., La Chine à Paris. Enquête au coeur d'un monde méconnu, Paris, Robert Laffont, 2012. ; Biao Xiang, Transcending boundaries: Zhejiangcun: The story of a migrant village in Beijing, Leiden, Brill Academic Pub, 2005. 2. Ya-Han Chuang, Anne-Christine Trémon, “Problematizing 'Chinatowns': conflicts and narratives surrounding chinese quarters in and around Paris", in Chinatowns Around the World: Gilded Ghetto, Ethnopolis, and Cultural Diaspora, 2013, pp. 187-214. 3. Dans cet article, nous utilisons le mot "wenzhou" comme un nom ou un adjectif marquant l'identité d'appartenir à une histoire migratoire commune, mais non le fait de naître à Wenzhou ou non. Par ailleurs, nous verrons plus tard dans le texte que de nombreux jeunes emploient le terme "Wen" pour se définir. 
parfois de manière contradictoire, par la trajectoire collective de la communauté d'origine ${ }^{5}$. Dans la continuité de ce modèle théorique, nous examinerons dans quelle mesure grandir dans une famille commerçante permet la transmission de valeurs, de préférences professionnelles, jusqu'à l'élargissement de l'entreprise familiale ou du réseau économique communautaire. Pour les jeunes éduqués en France, que signifie le fait de reprendre l'entreprise familiale de leurs parents?

Nous commencerons par observer comment les parents entrepreneurs perçoivent l'éducation supérieure. Nous exposerons la manière dont les origines sociales de ces jeunes influencent leurs choix professionnels et les stratégies afin de prolonger l'entreprise familiale. Cela nous conduira à envisager à quel point les pratiques familiales et communautaires épousent les récits culturalistes. Les jeunes ayant souvent travaillé
En plus des secteurs d'activité traditionnellement occupés par les migrants en Europe

- la restauration, le textile,

la maroquinerie, etc. -, ils ont

établi un énorme marché export qui compte plus de mille magasins dans la banlieue parisienne. de commerce de gros d'importdans les entreprises familiales tendent à percevoir l'intérêt personnel et l'intérêt familial ou communautaire comme inséparables, malgré des négociations parfois douloureuses entre les attentes familiales et les trajectoires personnelles en tant que left behind children ${ }^{6}$. Cela les pousse à poursuivre l'innovation du modèle économique de l'entreprise familiale afin d'améliorer l'image collective des commerçants wenzhou en France, souvent associés au stigmate des fabricants de produits bas de gamme. Enfin, nous montrerons que les entreprises familiales tendent à devenir une ressource identitaire en partie élaborée sur le récit d'une réussite économique motivée par un esprit d'entrepreneuriat proprement chinois.

\section{Une attitude pragmatique à l'égard de l'éducation supérieure}

Dans de nombreuses sociétés d'accueil, notamment aux États-Unis, les parents d'origine chinoise se font remarquer par la forte valorisation de l'éducation. Ce phénomène contribue à la formation d'une étiquette de "minorité modèle ${ }^{7 "}$. En comparaison, en France, l'existence d'une niche ethnique contribue à une attitude plus nuancée, voire instrumentaliste, des parents commerçants wenzhou à l'égard de léducation de leurs enfants. Ce pragmatisme résulte non seulement d'une méconnaissance de la place des étrangers dans le marché de l'emploi, mais aussi de la valorisation de l'indépendance ${ }^{8}$. Ayant connu une mutation rapide de position sociale, de paysans à entrepreneurs transnationaux ${ }^{9}$, les migrants wenzhou tendent à privilégier les bénéfices matériels par rapport au capital culturel, et ont ainsi un regard négatif sur l'investissement dans l'enseignement supérieur. Nous pouvons prendre l'exemple de Gao Fong, grossiste d'habits qui possède trois magasins situés dans divers quartiers de la région parisienne. Selon lui, "'eest bien de pouvoir faire des études et de trouver des jobs stables. Mais on connaît aussi des enfants d'un ami qui ont démissionné d'un travail à la banque. Si, après avoir fait toutes ces années d'études, ce n'est que pour gagner quelques milliers d'euros par mois,

\footnotetext{
4. Cet article s'appuie sur une série d'entretiens avec douze jeunes recrutés par les réseaux de l'Association des jeunes Chinois de France (voir à ce sujet le texte de Ken CHEN dans ce dossier). Les jeunes dont nous analysons les entretiens ici comptent huit hommes et quatre femmes. Ils sont nés entre 1988 et 1995. Hormis une employée d'une compagnie de production de cinéma, les autres travaillent tous dans le milieu économique chinois de la région parisienne. Cet article se nourrit du terrain réalisé dans le cadre de ma thèse intitulée "Migrants chinois à Paris : au-delà de l"intégration' : la formation politique d'une minorité" et soutenue le 25 novembre 2015 à l'université Paris-IV. 5. Alejandro Portes, Min Zhou, “The new second generation: segmented assimilation and its variants", in Annals of the American Academy of Political and Social Science, vol. 530, 1993, pp. 74-96. 6. Voir Simeng Wang, “'Enfant abandonné en Chine puis domestique en France? Qu'est-ce que je suis pour eux?!' Obligations familiales à rebours des enfants migrants d'origine chinoise à Paris", in Enfances, Familles, Générations, $n^{\circ} 20,2014$, pp. 21-44; ASIS, Maruja MB. “Living with migration: Experiences of left-behind children in the Philippines", in Asian Population Studies, 2006, vol. 2, no 1, p. 45-67. 7. Min Zhou,. "Are Asian Americans becoming 'White?"', in Contexts, 2004, vol. 3, no 1, 2004, pp. 29-37. 8. Claire Zalc, Melting-Shops. Une histoire des commerçants étrangers en France, Paris, Perrin, 2010. Voir notamment le chapitre 5. 9. Concernant leur trajectoire migratoire, voir Richard Beraha, Junliang Pan, Liwen Dong et al., op. cit. 10. Entretien réalisé le 18 mars 2014 dans sa boutique, Paris, $\mathrm{Xl}^{\mathrm{e}}$ arrondissement.
} 
cela ne me semble pas valoir le coup ${ }^{10 "}$. Étant l'un des grossistes les plus renommés de la communauté, les affaires familiales de Gao Fong sont réparties entre Paris, Guangzhou, San Francisco et Shanghai. Ses deux enfants ont choisi de reprendre l'entreprise familiale après avoir obtenu des masters dans le domaine du commerce international.

S'ajoute encore une méfiance à l'égard du marché de l'emploi qu'ils estiment discriminatoire envers les minorités ethnoraciales. Comme l'a déjà souligné Mohamed Madoui pour les entrepreneurs issus de familles maghrébines, devenir entrepreneur n'est pas toujours un choix motivé, c'est souvent une solution pour contourner le plafond de verre que subissent les minorités visibles ${ }^{11}$. "Chez les Wen- zhou de campagne, on dit très souvent une chose : telle ou telle personne, elle a fait des études, et, regarde, elle est revenue aider ses parents. Elle est redevenue vendeuse. Donc, à quoi ça sert de faire des études ${ }^{12}$ ?", explique Wan Jie, né en 1988 et diplômé du master de finance d'une université parisienne réputée dans le domaine du commerce international. Dans un marché de l'emploi de plus en plus compétitif pour les jeunes diplômés, l'éducation supérieure n'est plus une garantie d'ascension sociale. À ce titre, les parents wenzhou partagent un sentiment de désenchantement que connaissent déjà d'autres jeunes de parents immigrés ${ }^{13}$. Le modèle d'entreprise familiale fondé sur le travail collectif renforce également l'hostilité des parents immigrés à l'égard 
de l'éducation supérieure. Ayant une connaissance limitée de la langue, de l'administration ou de la loi française, les parents immigrés tendent à souhaiter que leurs enfants participent aux entreprises familiales après avoir

Plutôt qu'une obligation

familiale, pour les jeunes d'origine de Wenzhou ayant fait des études supérieures,

reprendre l'entreprise familiale se présente comme un compromis entre leur capital culturel et leurs attentes financières ou celles de leur famille. pouvais parler français, que ton français était suffisant pour faire du business, on pouvait arrêter l'école ${ }^{14}$." Plutôt qu'une obligation familiale, pour les jeunes d'origine de Wenzhou ayant fait des études supérieures, reprendre l'entreprise familiale se présente comme un compromis entre leur capital culturel et leurs attentes financières ou celles de leur famille. Qu'ils choisissent de reprendre les affaires de leurs parents ou non, tous évoquent l'importance des attentes familiales, des obligations morales ou de la transmission des valeurs dites "wenzhou" dans l'explication de leur parcours.

\section{Hériter, un choix rationnel pour concilier l'intérêt personnel et le bénéfice familial}

Entrer dans le marché de l'emploi représente un critère d'intégration important dans les études sur la migration ${ }^{15}$. Cependant, pour les jeunes issus de Wenzhou, poursuivre des études supérieures ne les incite pas toujours à s'intégrer dans le marché de l'emploi. Ayant grandi dans des familles de com- merçants, certains jeunes perçoivent l'entreprise familiale comme plus désirable, en termes de revenus, mais aussi de reconnaissance personnelle, que le travail salarié offert par les entreprises françaises. Le choix d'Hélène Zhang en est la meilleure illustration. Après six mois de travail en alternance, elle s'est rendu compte que les revenus n'étaient pas satisfaisants. Elle explique : "Je voyais mon chef, il travaillait le matin très tôt. Il faisait des horaires très longs. Je me suis dit, ça gagne pas assez en fait. Si j'aidais mes parents, au moins c'est l'entreprise familiale, et on gagne de l'argent pour toute la famille, c'est mieux ${ }^{16}$." Ainsi, elle a accepté de travailler dans le magasin de commerce de bazar à Aubervilliers après avoir obtenu un master 2 en biochimie.

De son côté, Estelle Gao, l'aînée de Gao Fong, considère qu'elle a plus d'opportunités d'épanouissement personnel dans l'entreprise familiale qu'en travaillant dans une entreprise française, après avoir eu une expérience frustrante dans la recherche d'un stage durant sa formation de master. Selon son père, "étant étranger, ce n'est pas facile de s'intégrer en France. Si ça l'intéresse de nous aider, pourquoi pas? Ce n'est pas plus mal de travailler entre nous". Aujourd'hui gérante de l'un des magasins de gros situé dans le quartier du Sentier, elle partage sa vie entre la Chine et Paris. Cette évolution rejoint la tendance observée chez les jeunes issus de Wenzhou en Italie. Ayant un capital culturel élevé, ils profitent de leurs compétences et contribuent à l'élargissement de l'entreprise familiale. En conséquence, les commerçants chinois tendent aujourd'hui à l'emporter sur les entreprises italiennes traditionnelles et deviennent des acteurs importants de l'industrie de la mode ${ }^{17}$.

Ainsi, rejoindre l'entreprise familiale offre non seulement plus de possibilités d'épanouissement personnel, mais aussi plus de bénéfices pour l'intérêt familial, perçu comme inséparable de l'intérêt personnel ${ }^{18}$. À ce titre, Wan Jie explique 
comment l'intérêt familial le préoccupe depuis son enfance: "Quand jétais petit, ma mère faisait de la confection à la maison, je l'aidais à retourner les vêtements. Après, mes parents ont eu un très petit restaurant à Belleville, et ensuite ils sont devenus grossistes. Je travaille toujours à leurs côtés, j’ai jamais pensé que cétait 'aider'. On est une famille, leur argent est mon argent, et vice versa. On ne fait pas de différence ${ }^{19}$." C'est aussi un tel sens des responsabilités familiales qui l'a fait rester travailler auprès de ses parents après ses études supérieures. Dans un premier temps, il a aidé ses parents à gérer leur entreprise de commerce de gros de chaussures qui était au bord de la faillite à cette époque. L'entreprise ayant été liquidée en 2010, Wan Jie a ensuite utilisé le capital familial pour ouvrir un supermarché franchisé avec sept salariés, incluant ses deux parents. Il s'agit, selon lui, d'une solution viable permettant à la fois de satisfaire les attentes communautaires de statut social et de s'occuper de ses parents. "Je pense que je ne vais pas faire un travail par passion. Parce que je vis dans un milieu où l'argent est très important pour gagner la reconnaissance $^{20}$." L'attente familiale, voire communautaire, de la performance économique devient un élément crucial de réalisation de soi. L'expérience d'avoir aidé ses parents à travailler dans l'atelier, et ensuite dans le magasin familial, semble contribuer à cette perception.

\section{Innovation, une nécessité dans un marché ethnique saturé}

L’existence d'un marché ethnique spécialisé dans l'import-export fournit une option valorisante pour les jeunes issus de familles d'entrepreneurs. Or l'accroissement du nombre de commerçants grossistes et l'augmentation du coût de production en Chine affaiblissent la marge des grossistes. Face à un marché de plus en plus concurrentiel, les

jeunes ne peuvent plus se contenter d'hériter du capital et du savoir-faire de leurs parents. Il s'avère nécessaire pour eux de trouver des pistes d'innovation permettant d'améliorer le modèle économique de la génération précédente ${ }^{21}$.

Une stratégie dominante consiste à monter en gamme en cherchant à créer sa propre marque. C'est ainsi la mission que confie Gao Fong à sa fille Estelle qui gère aujourd'hui la boutique familiale du Sentier et vise à monter en gamme afin de s'installer aux Galeries Lafayettes plus tard. Habile à communiquer avec les clients francophones, elle prend en charge le marketing visant les clients français ou européens. Cela permet à Gao Shan de se concentrer sur la gestion de la chaîne de production en Chine. Il affirme: "Ma génération est trop vieille. Nous n'avons ni l'instinct pour les tendances, ni la compétence linguistique et culturelle pour travailler avec les clients français. Au contraire, nos enfants sont formés ici et ont des compétences pour travailler ainsi. Comme ils ont le courage de tenter, je suis ravi de les laisser se battre sur le front, et, moi, j'assure les arrières 22 ."

Une autre stratégie consiste à employer leurs connaissances pour trouver des voies permettant d'élargir la clien-

Il y a fort à parier que la réussite d'entrepreneurs originaires de Wenzhou ne reposera plus uniquement sur le marché ethnique traditionnel enclavé, mais s'inscrira plus largement dans un monde économique intégré. tèle. Ainsi, l'Internet, nouvelle plate-forme de vente, devient un terrain attractif pour les jeunes. On peut à ce titre citer le parcours d'Arnaud Zhou, responsable d'une entreprise de vente en ligne pour les grossistes. Né en 1988 dans la région parisienne et titulaire d'un master en développement Web, il lance en 2011 avec deux amis, également originaires de Wenzhou, un nouveau site de commerce en ligne qui permet aux commerçants de détail dans 36 pays européens, jusqu'à l'Islande et l'Ukraine, d'entrer en contact avec les grossistes chinois à Paris. "L'Internet peut attirer des clients qui habitent loin, qui achètent en 
petites quantités et qui ne disposent pas du budget ou du personnel pour se déplacer jusqu'ici. Mais une fois que la relation de confiance s'installe, leurs quantités d'achats pourraient s'accroître ${ }^{23}$." À la différence des autres exemples cités ci-dessus, Arnaud n'est pas un "héritier" au sens matériel. Ses parents, restaurateurs à Montreuil, "ont beau être de Wenzhou", il n'a pas vécu dans une atmosphère communautaire et a peu d'amis de la même origine ethnique. Toutefois, il n'hésite pas à souligner l'influence de la tradition entrepreneuriale de Wenzhou en introduisant son parcours: "Dans la culture wen, mes parents m'ont toujours poussé à créer quelque chose, à avoir un bon cadre de vie. Que je sois chef, avocat, cadre, etc., avoir une bonne qualité de vie est important $t^{24}$."

En mettant l'accent sur l'éducation familiale, Arnaud montre à quel point son origine sociale l'incite à poursuivre une position sociale élevée en devenant son propre maitre. À travers ce cas, on peut remarquer que l'expérience de grandir dans une famille de commerçants favorise souvent un esprit d'entrepreneuriat. C'est pourquoi de plus en plus de jeunes de Wenzhou diplômés choisissent un métier indépendant tout en admettant avoir hérité d'une culture propre aux migrants origininaires de Wenzhou. Qu'ils reprennent l'entreprise familiale fondée par leurs parents ou qu'ils créent leur propre entreprise, leur capital culturel plus élevé les incite à sortir du modèle traditionnel des petites entreprises familiales. Ils poursuivent souvent une démarche plus rationnelle et modernisée. Ainsi, lorsque de plus en plus de jeunes de Wenzhou travailleront à leur compte, nous assisterons sûrement à une reconfiguration du marché communautaire. La taille de celui-ci se réduira tout en montant en gamme. Il y a fort à parier que la réussite d'entrepreneurs originaires de Wenzhou ne reposera plus uniquement sur le marché ethnique traditionnel enclavé, mais s'inscrira plus largement dans un monde économique intégré.

Chérif Haïdara 29 ans, franco-malien, a passé 7 années en Chine où il a appris le mandarin et suivi des études de commerce. Il est aujourd'hui acheteur régulier dans le "triangle d'or" à Aubervilliers, mai 2016. (c) Camille Millerand

\section{Quand un récit identitaire se greffe sur un esprit d'entrepreneuriat}

Bien que les parents entrepreneurs tendent à avoir un regard négatif quant à l'investissement dans les études supérieures de leurs enfants, tout le monde ne peut pas hériter d'une entreprise familiale viable dans un marché quasiment saturé. Ainsi, de nombreux jeunes choisissent de poursuivre une formation supérieure afin d'entrer dans le marché de l'emploi. Pour autant, sortir de l'économie ethnique ne conduit pas à l'abandon de l'appartenance à une communauté commerçante délimitée. Nombreux sont ceux parmi ces jeunes qui valorisent une indépendance professionnelle et souhaitent devenir leur propre chef. Cela conduit une grande partie des jeunes à privilégier une formation juridique, d'expertise comptable ou de médecine, qui mène 
à une profession libérale. Tel est le choix de Thomas $\mathrm{Du}$, avocat en droit commercial, qui a ouvert son cabinet en 2009. Ayant toujours été intéressé par les études en sciences sociales, il décide très tôt de faire des études de droit afin de concilier sa passion intellectuelle et son envie de travailler à son propre compte. "On m'a toujours dit que, pour réussir, il faut être son propre patron, j'ai donc choisi la profession d'avocat parce que c'est un métier où je peux concilier mes études et le caractère indépendant d'un métier libéral25." Outre le désir d'être indépendant, l'envie de travailler à son propre compte l'incite aussi à ouvrir son propre cabinet très tôt dans sa carrière. Ayant travaillé seulement trois mois en collaboration avec un avocat français, il lance son cabinet d'avocat dans un quartier ayant une forte concentration de commerces chinois. Ce choix lui permet de bénéficier d'une clientèle provenant des réseaux communautaires. Il affirme: "Tout ce qui concerne la clientèle, c'est surtout du bouche-à-oreille. Dès le début, j'ai déjà un portefeuille de clients car il y a pas mal de personnes autour de moi qui ont besoin d'un avocat pour pouvoir acheter un restaurant, acheter un magasin, etc. ${ }^{26}$." Dans une communauté de commerçants migrants ayant des besoins continus de conseils juridiques, le lien communautaire octroie à Thomas Du une réserve de clientèle. Sans avoir hérité d'un capital financier, son origine lui offre un capital social considérable pour la réussite professionnelle. Par ailleurs, il est frappant de voir la façon dont les jeunes conçoivent l'"esprit capitaliste" comme un attribut inhérent à la population wenzhou. En effet, quel que soit leur métier, les jeunes qualifient unanimement le talent commercial et le travail acharné comme des caractéristiques acquises par leur éducation familiale. Comme cela a été indiqué par Mathieu Cai, né en 1994 de deux parents qui tenaient un grand restaurant dans une banlieue parisienne et diplômé d'un master d'une école de commerce prestigieuse à Londres: "Je pense que dans n’importe quelle situation, je n'aurai pas de problèmes. Parce que j'ai travaillé toute ma jeunesse dans le restaurant, donc ça m'a forcé à être en contact avec

les autres. Je suis vraiment adaptable. Et ça, je sais que je le tire de ma culture chinoise, en fait ${ }^{27}$."

À cette socialisation précoce dans le restaurant familial s'ajoute encore un sentiment d'appartenance à la diaspora wenzhou, fortement caractérisée par sa visibilité dans le milieu commercial. Mathieu décrit la façon dont la présence remarquable des commerçants wenzhou dans le monde des entreprises chinoises l'incite à poursuivre des études dans les meilleures écoles de commerce du monde : "Pour le master, je vise les meilleures écoles de commerce dans le monde. Parce que je me dis que, chez les Wen, on a ce sens des affaires. On a beaucoup de capitaux, on a de l'argent. Et ce qui nous manque, c'est le côté intellectuel. On manque de représentation dans ce monde. Ça serait bien quion se présente dans ce monde

De la reprise du magasin familial au désir de renouveler l'image des commerçants de Wenzhou, en passant par la conscience d'une nécessité d'actualiser le modèle économique, on observe un large éventail de formes de transmission intergénérationnelle dans la famille commerçante immigrée. aussi, pour montrer que les Wen ne sont pas que des gens qui s'occupent de restaurants et de commerces $^{28}$." La réussite économique de ces migrants, fondée principalement sur le modèle de l'entreprise familiale, épouse ici un récit identitaire collectif poussant les jeunes à poursuivre un capital culturel plus élevé afin de faire évoluer l'image collective de cette communauté commerçante.

\section{La Chine mondialisée, une nouvelle ressource identitaire transmissible}

De la reprise du magasin familial au désir de renouveler l'image des commerçants de Wenzhou, en passant par la conscience d'une nécessité d'actualiser le modèle économique, on observe un large éventail de formes de transmission intergénérationnelle dans la famille commerçante immigrée. Le passage de la valorisation de la ressource matérielle à celle de la croyance symbolique atteste le 
rôle changeant de la Chine. Au fur et à mesure que le marché chinois connaît la croissance, le lien matériel ou symbolique avec la Chine devient indispensable pour que ces jeunes se sentent légitimes en tant que descendants de cette communauté commerçante et aient envie de mettre en valeur leur héritage.

D'un côté, un nombre croissant de parents entrepreneurs désirent envoyer leurs enfants étudier en Chine afin de former le futur héritier de l'entreprise familiale. Comme cela a été confié par un grossiste d'habits : "Mon fils a grandi ici, donc sa manière de penser ressemble plus à celle des Français qu'à celle des Chinois. Il est trop direct ${ }^{29}$." Afin que son fils puisse assimiler la "pensée chinoise", qui est selon lui "plus subtile et plus sophistiquée", il a envoyé son fils étudier en Chine après le bac durant trois ans. Aujourd'hui, ce dernier gère le magasin familial et s'occupe de la communication avec plusieurs entreprises de fabrication à Guangzhou. Puisque le commerce des entrepreneurs de Wenzhou à Paris dépend largement de la production en Chine, il s'avère nécessaire pour les jeunes générations d'approfondir leur connaissance linguistique et culturelle de la Chine avant de reprendre le magasin familial.

D'un autre côté, l'image contemporaine de la Chine mondialisée, synonyme de cosmopolitisme, devient une icône séduisante dont les jeunes Franco-Chinois souhaitent se rapprocher. Ainsi, Chloé Cai, sœur aînée de Mathieu Cai cité plus haut, avoue qu'elle a tenté de plaire à ses parents en allant travailler en Chine, même si elle a refusé leur demande de reprendre le restaurant familial : "Je suis toujours très proche de ma famille. Cela m'embêtait de ne pas les satisfaire, parce que j'ai toujours su qu'ils avaient travaillé très dur pour la famille. Et face au fait qu’ils soient déçus par moi, j’ai gardé au fond de moi cette culpabilité très forte. Donc, à l’âge de 20 ans, je me suis dit que j'allais essayer de me rapprocher de mes parents, et la seule façon, cétait de tenter mes expériences en Chine $e^{30}$." Pour son frère Mathieu, ses expériences de voyages en Chine servent à réaffirmer son appartenance à une diaspora chinoise. Il raconte: "En 2008, en BTS, j'ai fait un stage de trois mois et demi à Shanghai. C'était génial. J'ai rencontré des Chinois comme moi, qui sont des Chinois de Suède, des Chinois de Hollande, des Chinois de Bolivie, des Chinois d'Afrique, et on a tous cet esprit commun chinois. On avait beaucoup de débats intéressants sur l'avenir, sur le fait de savoir ce que c'est qu'être chinois? Entre les gens de Wenzhou, on sait ce que sont les choses importantes. L'argent, c'est le meilleur ${ }^{31}$." Cette équation entre son appartenance wenzhou et un héritage commun de la diaspora chinoise est frappante. Elle montre à quel point les valeurs entrepreneuriales transmises au sein des familles commerçantes sont associées à l'image moderne d'une Chine développée, fournissant ainsi une ressource identitaire qui articule les expériences migratoires, l'esprit capitaliste et le sentiment d'appartenant à une même entité culturelle.

\section{Conclusion}

Cet article examine l'influence de la trajectoire collective de la communauté des entrepreneurs de Wenzhou en France sur les choix professionnels de leurs descendants. À la différence d'un paradigme classique d'intégration supposant une "convergence individuelle des caractéristiques des migrants et de leurs descendants vers celles des natifs'32", les témoignages de ces jeunes illustrent plutôt la théorie de l'assimilation segmentée, c'est-à-dire une trajectoire privilégiant une intégration économique dont le cadre est constitué par les expériences migratoires des parents, sans que pour cela d'autres formes d'intégration soient déterminées de façon mécanique. En effet, malgré des négociations parfois conflictuelles au sein de la famille, grandir dans une famille d'entrepreneurs migrants détermine considérablement les choix professionnels de la jeune génération. Cela joue également sur 
l'équilibre adopté entre l'épanouissement personnel et l'intérêt familial. Pour les jeunes diplômés, l'économie ethnique fournit la possibilité d'obtenir des revenus élevés sans passer par le mode de recrutement classique des entreprises françaises. Les valeurs capitalistes transmises au sein de ces familles commerçantes ainsi que l'image d'une Chine au centre de l'économie mondiale sont la base d'un récit identitaire, qui n'est pas toujours transmis avec aisance au sein de la famille ${ }^{33}$, mais qui permet aux jeunes de fondre leur expérience personnelle avec celle d'une Chine triomphante ${ }^{34}$. Par conséquent, leur réussite n'est plus une simple trajectoire personnelle, mais le devenir économique de toute une diaspora.

33. Voir Estelle Auguin, "Le lien filial en migration. Les Chinois du sud du Zhejiang", Hommes \& Migrations, $n^{\circ}$ 1254, 2005, pp.18-29. 34. Donald M. Nonini, Aihwa Ong, "Chinese transnationalism as an alternative modernity", in Ungrounded empires. The Cultural Politics of Modern Chinese Transnationalism, London, Routledge, 1996.

\section{La Marcheuse Film français de Naël Marandin (2016)}

La Marcheuse, premier long métrage du réalisateur Naël Marandin, est une fiction inspirée de la vie des prostituées chinoises à Paris. Aiyu, l'héroïne, est originaire du Dongbei, province au Nord de la Chine massivement touchée par les fermetures d'usine, qu'elle décide de quitter après son licenciement et son divorce. Elle paye son billet pour Paris - et s'endette auprès de passeurs -, pensant y gagner sa vie. Sans papier, elle est d'abord nounou dans une famille chinoise, puis aide à domicile chez une personne âgée. Elle complète son salaire en offrant des services sexuels sur le boulevard de la Villette, y trouvant une plus grande liberté qu'en étant exploitées par des familles du Wenzhou - principale origine des immigrés chinois de Paris et majoritairement commerçants. Dans ce film, l'illusion d'une grande communauté chinoise est démystifiée, sans annihiler les relations de solidarité qui se créent non pas entre "les Chinois" mais entre des femmes qui partagent des trajectoires et un quotidien similaires. Ce quotidien est incarné dans les nombreuses scènes de rue, Aiyu au premier plan : les clients qui errent sur le boulevard l'œil hagard, les arrestations hasardeuses des femmes qui "marchent", les tireurs de sac qui ont flairé les victimes faciles, les groupes de femmes qui discutent au carrefour d'une rue, tout en restant attentives aux violences alentours dont elles sont potentiellement la cible. Aiyu et ses amies ne sont pas présentées dans le film comme des "victimes" ou des "pauvres filles" mais comme des femmes qui ont fait et continuent à faire des choix, malgré les obstacles qu'elles rencontrent. Violence comme silence, suggérés dans le film sans en être le centre, sont présentés comme des conséquences non pas de la prostitution mais de leur situation irrégulière et des discriminations à la fois interpersonnelles et institutionnelles. Montrer ces images, c'est ouvrir et inciter à ouvrir des espaces de parole pour écouter ces femmes et réfléchir à la réalité complexe de la prostitution. Le film sort en DVD en juillet 2016. 